Modern Physics Letters A

Vol. 32, No. 40 (2017) 1799001 (18 pages)

(C) World Scientific Publishing Company

DOI: $10.1142 / \mathrm{S} 0217732317990012$

\title{
Author index Volume 32
}

Aaltonen, T., et al. (CDF Collab.), Observation of the $Y(4140)$ structure in the $J / \psi \phi$ mass spectrum in $B^{ \pm} \rightarrow J / \psi \phi K^{ \pm}$decays

Abdalla, E., see Lin

Abeysekera, B., see Matei

Abreu, E. M. C. \& Godinho, C. F. L., Gauge invariant actions for the noncommutative phase-space relativistic particle

Abreu, E. M. C., Fernandes, R. L., Mendes, A. C. R., Neto, J. A. \& Neves, M. Jr., Duality and gauge invariance of non-commutative spacetime Podolsky electromagnetic theory

Abreu, E. M. C., see Moradpour

Abrunhosa, A. J. , see Alves

Accioly, A. \& Herdy, W., Higherorder gravity and the classical equivalence principle

Accioly, A., Correia, G., de Brito, G. P., de Almeida, J. \& Herdy, W., Relating renormalizability of $D$ dimensional higher-order electromagnetic and gravitational models to the classical potential at the origin

Acikgoz, I., see Keskin

Addazi, A. \& Khlopov, M. Yu., Dark matter from Starobinsky supergravity

Addazi, A., Limiting first-order phase transitions in dark gauge sectors from gravitational waves experiments

Addazi, A., Weak gravity conjecture as a razor criterium for exotic $D$ brane instantons

Afonin, S. S., A collisional model for light scalar mesons

Afonin, S. S., Meson spectrum for arbitrary twist in soft-wall holographic model

32 (2017) 1750139

32 (2017) 1750134

32 (2017) 1740011

Ahmad, M., see Shamir

Ahmad, Z., see Khan

Ahmedov, B., see Toshmatov

Ajaltouni, Z. J., see Di Salvo

Akhtar, S. S., see Khan

Akiyama, T., see Kawamura

Alanne, T., Rzehak, H., Sannino, F. \& Thomsen, A. E., Raising the SUSY-breaking scale in a Goldstone-Higgs model

Alencar, G., see Landim

Alexander, H., da Paz, I. G. \& Samtwo scalar fields in an expanding spacetime

32 (2017) 1750078

32 (2017) 1740013

32 (2017) 1750185

32 (2017) 1750048

32 (2017) 1750182

32 (2017) 1740002

32 (2017) 1750049

Ambjørn, J. \& Watabiki, Y., A modified Friedmann equation

Amerio, S., see Aaltonen

32 (2017) 1750014

Amidei, D., see Aaltonen

Amirabi, Z., see Mazharimousavi

32 (2017) 1750179 M. \& Bhatti, A. S., Comparison study of the $p_{T}$ distributions of the charged particles in $p-P b$ interactions at LHC energies

Alimohammadi, M., see Hassanabadi

Allen, R. E. \& Saha, A., Dark matter candidate with well-defined mass and couplings

Álvarez González, B., see Aaltonen

Alves, F., Alves, V. H. P., Do Carmo, S. J. C., Neves, A. C. B., Silva, M. \& Abrunhosa, A. J., Production of copper-64 and gallium- 68 with a medical cyclotron using liquid targets

Anastassov, A., see Aaltonen paio, M., Entanglement between

32 (2017) 1750155

32 (2017) 1750086

32 (2017) 1750192

32 (2017) 1775001

32 (2017) 1750043

32 (2017) 1750124

32 (2017) 1750058

32 (2017) 1750143

32 (2017) 1750193

32 (2017) 1750104

Andrighetto, A., see Maggiore
32 (2017) 1750167

32 (2017) 1750085

32 (2017) 1730022

32 (2017) 1750139

32 (2017) 1740013

32 (2017) 1740013

32 (2017) 1750224

32 (2017) 1750139

32 (2017) 1750139

32 (2017) 1750064

32 (2017) 1750139

32 (2017) 1740010 
Anisovich, V. V., Matveev, M. A., Nyiri, J. \& Semenova, A. N., Narrow pentaquarks as diquarkdiquark-antiquark systems

Annovi, A., see Aaltonen

Antonini, P., see Maggiore

Antos, J., see Aaltonen

Anwar, A., see Zaeem-ul-Haq Bhatti

Apollinari, G., see Aaltonen

Apostolopoulos, P. S., Intrinsic conformal symmetries in Szekeres models

Appel, J. A., see Aaltonen

Apresyan, A., see Aaltonen

Arias, P., Das, A. K., Gamboa, J. \& Méndez, F., Generalized Dirac duality and $\mathrm{CP}$ violation in a twophoton theory

Arisawa, T., see Aaltonen

Arraut, I. \& Chelabi, K., Vacuum degeneracy in massive gravity: Multiplicity of fundamental scales Artikov, A., see Aaltonen

Artun, O., A study of nuclear structure for ${ }^{244} \mathrm{Cm},{ }^{241} \mathrm{Am},{ }^{238} \mathrm{Pu}$, ${ }^{210} \mathrm{Po},{ }^{147} \mathrm{Pm},{ }^{137} \mathrm{Cs},{ }^{90} \mathrm{Sr}$ and ${ }^{63} \mathrm{Ni}$ nuclei used in nuclear battery

Asaadi, J., see Aaltonen

Asada, H., Gravitational lensing by exotic objects

Ashmanskas, W., see Aaltonen

Ashraf, S., see Zaeem-ul-Haq Bhatti

Askin, M., Salti, M. \& Aydogdu, O., Cosmology via thermodynamics of polytropic gas

Asorey, M., Ciaglia, F. M., Di Cosmo, F. \& Ibort, A., Covariant brackets for particles and fields

Asorey, M., Ciaglia, F. M., Di Cosmo, F., Ibort, A. \& Marmo, G., Covariant Jacobi brackets for test particles

Asorey, M., Ciaglia, M., Di Cosmo, F. \& Ibort, A., Covariant brackets for particles and fields

Atiyah, M. F., Geometric models of Helium

Auerbach, B., see Aaltonen

Aurisano, A., see Aaltonen

Avetisyan, A. S. \& Khurshudyan, As. Zh., Green's function approach in approximate controllability for nonlinear physical processes

Aydogdu, O., see Askin

Aydogdu, O., see Salti
Aygün, S., see Sahoo

Azadifar, R. \& Mahdavi, M., Power deposition of deuteron beam in

32 (2017) 1750154

32 (2017) 1750139

32 (2017) 1740010

32 (2017) 1750139

32 (2017) 1750111

32 (2017) 1750139

32 (2017) 1750099

32 (2017) 1750139

32 (2017) 1750139

32 (2017) 1750032

32 (2017) 1750139

32 (2017) 1750112

32 (2017) 1750139

32 (2017) 1750117

32 (2017) 1750139

32 (2017) 1730031

32 (2017) 1750139

32 (2017) 1750111

32 (2017) 1750177

32 1792002(E)

32 (2017) 1750122

32 (2017) 1750100

32 (2017) 1750079

32 (2017) 1750139

32 (2017) 1750139

32 (2017) 1730015

32 (2017) 1750177

32 (2017) 1750183 fast ignition

Azfar, F., see Aaltonen

Badgett, W., see Aaltonen

Bakhshi, Z., Panahi, H. \& Golchehre, S. G., Four-dimensional quantum oscillator and magnetic monopole with $\mathrm{U}(1)$ dynamical group

Balachandran, A. P., Momen, A. \& de Queiroz, A. R., Equations of motion as covariant Gauss law: The Maxwell-Chern-Simons case

Balart, L. \& Fernando, S., A Smarr formula for charged black holes in nonlinear electrodynamics

Bamba, K., Odintsov, S. D. \& Saridakis, E. N., Inflationary cosmology in unimodular $F(T)$ gravity

Bao, Q. \& Qiang, L.-E, Null tests of nonlocal gravity with multi-axis gravity gradiometers in elliptic orbits: A theoretical study

Barbaro-Galtieri, A., see Aaltonen

Barnes, V. E., see Aaltonen

Barnett, B. A., see Aaltonen

Barria, P., see Aaltonen

Barrie, N. D. \& Kobakhidze, A., Generating luminous and dark matter during inflation

Bartos, P., see Aaltonen

Batalin, I. A. \& Lavrov, P. M., Physical quantities and arbitrariness in resolving quantum master equation

Bauce, M., see Aaltonen

Bauer, G., see Aaltonen

Bayrak, O., see Koyuncu

Bazeia, D. \& Bemfica, F. S., From scalar field theories to supersymmetric quantum mechanics

Bedeschi, F., see Aaltonen

Beecher, D., see Aaltonen

Beesham, A., see Sepehri

Behari, S., see Aaltonen

Bellettini, G., see Aaltonen

Bellinger, J., see Aaltonen

Belotsky, K., Rubin, S. \& Svadkovsky, I., Extended micro objects as dark matter particles

Belyaev, I., Berezhnoy, A. V., Likhoded, A. K. \& Luchinsky, A. V., Comments on "Study of $J / \psi$ production in jets"

Bemfica, F. S., see Bazeia
32 (2017) 1750105

32 (2017) 1750016

32 (2017) 1750139

32 (2017) 1750139

32 (2017) 1750161

32 (2017) 1750133

32 (2017) 1750219

32 (2017) 1750114

32 (2017) 1750131

32 (2017) 1750139

32 (2017) 1750139

32 (2017) 1750139

32 (2017) 1750139

32 (2017) 1750087

32 (2017) 1750139

32 (2017) 1750068

32 (2017) 1750139

32 (2017) 1750139

32 (2017) 1750050

32 (2017) 1750073

32 (2017) 1750139

32 (2017) 1750139

32 (2017) 1750123

32 (2017) 1750139

32 (2017) 1750139

32 (2017) 1750139

32 (2017) 1740008

32 (2017) 1771002 32 (2017) 1750073 
Benchikha, A., Merad, M. \& Birkandan, T., Energy-dependent harmonic oscillator in noncommutative space

Bendjoudi, A. \& Mebarki, N., The quantum pentahedra

Benjamin, D., see Aaltonen

Beretvas, A., see Aaltonen

Berezhnoy, A. V., see Belyaev

Bezerra, V. B., Cunha, M. S., Freitas, L. F. F., Muniz, C. R. \& Tahim, M. O., Casimir effect in the Kerr spacetime with quintessence

Bhar, P., see Singh

Bhattacharya, S. \& Shankaranarayanan, S., Black hole event horizons - Teleology and predictivity

Bhatti, A. S., see Ali

Bhatti, A., see Aaltonen

Bhatti, M. Z., Yousaf, Z. \& Hanif, S., Stability of anisotropic compact objects in $f(T)$ gravity

Bhatti, M. Z., see Yousaf

Binbay, F., see Salti

Binkley, M., see Aaltonen

Birkandan, T., see Benchikha

Bisello, D., see Aaltonen

Bishi, B. K., see Sahoo

Bisschoff, B., Dominguez, C. A. \& Hernandez, L. A., Electromagnetic proton form factors in dual large$N_{c}$ QCD: An update

Biswas, G. \& Modak, B., Wormhole in 5D Kaluza-Klein cosmology

Bizjak, I., see Aaltonen

Bland, K. R., see Aaltonen

Blumenfeld, B., see Aaltonen

Bocci, A., see Aaltonen

Bodek, A., see Aaltonen

Böhmer, C. G. \& Hogan, P. A., A Vaidya-type generalization of Kerr spacetime

Bondar, A. E., Mizuk, R. V. \& Voloshin, M. B., Bottomoniumlike states: Physics case for energy scan above the $B \bar{B}$ threshold at Belle-II

Bortoletto, D., see Aaltonen

Boudreau, J., see Aaltonen

Boveia, A., see Aaltonen

Braccini, S. \& Scampoli, P., Preface

Brau, B., see Aaltonen

Brevik, I., see Normann

Brigliadori, L., see Aaltonen

Brisuda, A., see Aaltonen

Britto, A. L. M., Das, A. K. \& Frenkel, J., Fermion propagator in

32 (2017) 1750106

32 (2017) 1750015

32 (2017) 1750139

32 (2017) 1750139

32 (2017) 1771002

32 (2017) 1750005

32 (2017) 1750093

32 (2017) 1750186

32 (2017) 1750167

32 (2017) 1750139

32 (2017) 1750042

32 (2017) 1750163

32 (2017) 1750183

32 (2017) 1750139

32 (2017) 1750106

32 (2017) 1750139

32 (2017) 1750105

32 (2017) 1750036

32 (2017) 1750023

32 (2017) 1750139

32 (2017) 1750139

32 (2017) 1750139

32 (2017) 1750139

32 (2017) 1750139

32 (2017) 1750189

32 (2017) 1750025

32 (2017) 1750139

32 (2017) 1750139

32 (2017) 1750139

32 (2017) 1702002

32 (2017) 1750139

32 (2017) 1750026

32 (2017) 1750139 an external potential and generalized Airy functions

Bromberg, C., see Aaltonen

Brucken, E., see Aaltonen

Bucciantonio, M., see Aaltonen

Budagov, J., see Aaltonen

Budd, H. S., see Aaltonen

Budd, S., see Aaltonen

Burkett, K., see Aaltonen

Busetto, G., see Aaltonen

Bussey, P., see Aaltonen

Buzatu, A., see Aaltonen

Calabretta, L., Calanna, A., Cuttone, G., D'Agostino, G., Rifuggiato, D. \& Russo, A. D., Overview of the future upgrade of the INFN-LNS superconducting cyclotron

Calancha, C., see Aaltonen

Calandrino, R., Radioprotection guidelines for the safe use and decommissioning of a medical cyclotron

Calanna, A., see Calabretta

Camarda, S., see Aaltonen

Campanelli, M., see Aaltonen

Campbell, M., see Aaltonen

Campo, D., see Maggiore

Canelli, F., see Aaltonen

Canepa, A., see Aaltonen

Carls, B., see Aaltonen

Carlsmith, D., see Aaltonen

Carosi, R., see Aaltonen

Carrillo, S., see Aaltonen

Carron, S., see Aaltonen

Casal, B., see Aaltonen

Castellanos, E. \& Escamilla-Rivera, C., Modified uncertainty principle from the free expansion of a BoseEinstein condensate

Castro, A., see Aaltonen

Catastini, P., see Aaltonen

Cauz, D., see Aaltonen

Cavalcanti, R. T., see Hoff da Silva

Cavaliere, V., see Aaltonen

Cavalli-Sforza, M., see Aaltonen

Cerri, A., see Aaltonen

Cerrito, L., see Aaltonen

Cesarini, F., see Cicoria

Chakrabarty, S., see Das

Chakraborty, K., Rahaman, F. \& Mallick, A., A relativistic twofluid model of compact stars

Chakraborty, K., see Rahaman
32 (2017) 1750139

32 (2017) 1750171

32 (2017) 1750139

32 (2017) 1750139

32 (2017) 1750139

32 (2017) 1750139

32 (2017) 1750139

32 (2017) 1750139

32 (2017) 1750139

32 (2017) 1750139

32 (2017) 1750139

32 (2017) 1750139

32 (2017) 1740009

32 (2017) 1750139

32 (2017) 1740015 32 (2017) 1740009

32 (2017) 1750139

32 (2017) 1750139

32 (2017) 1750139

32 (2017) 1740010

32 (2017) 1750139

32 (2017) 1750139

32 (2017) 1750139

32 (2017) 1750139

32 (2017) 1750139

32 (2017) 1750139

32 (2017) 1750139

32 (2017) 1750139

32 (2017) 1750007

32 (2017) 1750139

32 (2017) 1750139

32 (2017) 1750139

32 (2017) 1730032

32 (2017) 1750139

32 (2017) 1750139

32 (2017) 1750139

32 (2017) 1750139

32 (2017) 1740014

32 (2017) 1750180

32 (2017) 1750055

32 (2017) 1750053 
Chan, A. H., see Zhang

Channey, K. S. \& Kumar, S., Phenomenological implications of two simple modifications to TriBimaximal mixing

Chelabi, K., see Arraut

Chen, J., see He

Chen, Y. C., see Aaltonen

Chertok, M., see Aaltonen

Chervon, S. V., see Fomin

Chervon, S. V., see Maharaj

Cheung, I. P., see $\mathrm{Ni}$

Chizhov, V. M., see Kirilova

Chkareuli, J. L. \& Kepuladze, Z., Emergent Yang-Mills theories from universal extra dimensions

Chlachidze, G., see Aaltonen

Chlebana, F., see Aaltonen

Cho, G.-C. \& Shimo, H., Search for lepton flavor violation at future lepton colliders

Cho, K., see Aaltonen

Choiński, J., see Szkliniarz

Chokheli, D., see Aaltonen

Chou, J. P., see Aaltonen

Chubykalo, A., Espinoza, A., Gonzalez-Sanchez, A. \& Gutiérrez Rodríguez, A., On the violation of the invariance of the light speed in theoretical investigations

Chung, W. H., see Aaltonen

Chung, W. S. \& Hassanabadi, H., Quantum mechanics on (anti)-de Sitter background

Chung, W. S., Generalized higherorder Snyder model

Chung, W. S., Parity-dependent noncommutative quantum mechanics

Chung, Y. S., see Aaltonen

Ciaglia, F. M., see Asorey

Ciaglia, F. M., see Asorey

Ciaglia, M., see Asorey

Cicoria, G., Cesarini, F., Infantino, A., Vichi, S., Zagni, F. \& Marengo, M., Characterization of ${ }^{41} \mathrm{Ar}$ production in air at a PET cyclotron facility

Ciobanu, C. I., see Aaltonen

Ciocci, M. A., see Aaltonen

Clark, A., see Aaltonen

Compostella, G., see Aaltonen

Convery, M. E., see Aaltonen

Conway, J., see Aaltonen

Corbo, M., see Aaltonen

Cordelli, M., see Aaltonen

\begin{tabular}{|c|c|c|}
\hline \multirow[t]{4}{*}{32 (2017) 1750020} & Correia, G., see Accioly & 32 (2017) 1750048 \\
\hline & Costa Filho, R. N., see Landim & 32 (2017) 1750193 \\
\hline & Cotăescu, I. I., de Sitter geodesics & 32 (2017) 1750223 \\
\hline & Cox, C. A., see Aaltonen & $32(2017) 1750139$ \\
\hline 32 (2017) 1750137 & Cox, D. J., see Aaltonen & 32 (2017) 1750139 \\
\hline 32 (2017) 1750112 & Craciun, L., see Matei & $32(2017) 1740011$ \\
\hline 32 (2017) 1750052 & Crescioli, F., see Aaltonen & 32 (2017) 1750139 \\
\hline 32 (2017) 1750139 & Cuenca Almenar, C., see Aaltonen & 32 (2017) 1750139 \\
\hline 32 (2017) 1750139 & Cuevas, J., see Aaltonen & $32(2017) 1750139$ \\
\hline 32 (2017) 1750129 & Culbertson, R., see Aaltonen & 32 (2017) 1750139 \\
\hline 32 (2017) 1750164 & Cunha, M. S., see Bezerra & 32 (2017) 1750005 \\
\hline 32 (2017) 1730029 & Cuttone, G., see Calabretta & 32 (2017) 1740009 \\
\hline \multirow[t]{3}{*}{32 (2017) 1750187} & D’Agostino, G., see Calabretta & 32 (2017) 1740009 \\
\hline & d'Ascenzo, N., see Aaltonen & 32 (2017) 1750139 \\
\hline & d'Errico, M., see Aaltonen & 32 (2017) 1750139 \\
\hline 32 (2017) 1750029 & D’Onofrio, M., see Aaltonen & 32 (2017) 1750139 \\
\hline 32 (2017) 1750139 & da Paz, I. G., see Alexander & $32(2017) 1750104$ \\
\hline 32 (2017) 1750139 & $\begin{array}{l}\text { da Silva, J. G. \& Ulhoa, S. C., On } \\
\text { gravitational energy in conformal } \\
\text { teleparallel gravity }\end{array}$ & 32 (2017) 1750113 \\
\hline 32 (2017) 1750127 & Dagenhart, D., see Aaltonen & 32 (2017) 1750139 \\
\hline 32 (2017) 1750139 & Dariescu, C., see Dariescu & 32 (2017) 1750003 \\
\hline 32 (2017) 1740012 & Dariescu, C., see Dariescu & 32 (2017) 1750174 \\
\hline
\end{tabular}

32 (2017) 1750139

32 (2017) 1750139

32 (2017) 1730033

32 (2017) 1750139

32 (2017) 1750138

32 (2017) 1750098

32 (2017) 1750009

32 (2017) 1750139

32 1792002(E)

32 (2017) 1750122

32 (2017) 1750100

32 (2017) 1740014

32 (2017) 1750139

32 (2017) 1750139

32 (2017) 1750139

32 (2017) 1750139

32 (2017) 1750139

32 (2017) 1750139

32 (2017) 1750139

32 (2017) 1750139
Dariescu, M.-A. \& Dariescu, C., Mathieu functions for fermions generated in magnetar's corona

Dariescu, M.-A. \& Dariescu, C., Quantum cosmology on $(k=-1)$ Friedmann-Robertson-Walker Universe evolving from stiff matter era to the dust dominated one

Das, A. K., Gamboa, J., Méndez, F. \& Tapia, N., Testing dark matter with the anomalous magnetic moment in a dark matter quantum electrodynamics model

Das, A. K., see Arias

Das, A. K., see Britto

Das, A., Dave, S. S., De, S. \& Srivastava, A. M., Towards laboratory detection of topological vortices in superfluid phases of QCD

Das, S., Ghosh, S. \& Chakrabarty, S., Thomas-Fermi model in Rindler space

Dash, S., Nandi, B. K., Nayak, R., Pandey, A. K. \& Sett, P., Comprehending particle production at RHIC and LHC energies using global measurements

Daszkiewicz, M., The HenonHeiles system defined on Liealgebraically deformed Galilei spacetime

Datta, M., see Aaltonen
32 (2017) 1750174

32 (2017) 1750003

32 (2017) 1750175

32 (2017) 1750032

32 (2017) 1750171

32 (2017) 1750170

32 (2017) 1750180

32 (2017) 1750060

32 (2017) 1750075 32 (2017) 1750139 
Dave, S. S., see Das de Almeida, J., see Accioly de Barbaro, P., see Aaltonen de Brito, G. P., see Accioly De Cecco, S., see Aaltonen de Cruz Pérez, J., see Solà De Lorenzo, G., see Aaltonen de Queiroz, A. R., see Balachandran De, S., see Das

Debnath, P. S. \& Paul, B. C., Emergent universe model with dissipative effects

Dell'Orso, M., see Aaltonen

Deluca, C., see Aaltonen

Demirchian, H., Note on constants of motion in conformal mechanics associated with near horizon extremal Myers-Perry black holes

Demortier, L., see Aaltonen

Deng, J., see Aaltonen

Deninno, M., see Aaltonen

Dev, P. S. B., Mohapatra, R. N. \& Zhang, Y., Heavy right-handed neutrino dark matter in left-right models

Devecchi, F. P., see Ribas

Devecchi, F. P., see Ribas

Devoto, F., see Aaltonen

Di Canto, A., see Aaltonen

Di Cosmo, F., see Asorey

Di Cosmo, F., see Asorey

Di Cosmo, F., see Asorey

Di Ruzza, B., see Aaltonen

Di Salvo, E. \& Ajaltouni, Z. J., Searching for new physics in semileptonic baryon decays

Diachenko, M. M. \& Kholodov, R. I., Energy losses of positive and negative charged particles in electron gas

Dittmann, J. R., see Aaltonen

Do Carmo, S. J. C., see Alves

Dominguez, C. A., see Bisschoff

Donati, S., see Aaltonen

Dong, P., see Aaltonen

Donmez, O., Angular velocity perturbations inducing the PapaloizouPringle instability and QPOs in the torus around the black hole

Dorigo, M., see Aaltonen

Dorigo, T., see Aaltonen

dos Santos, R., see Pereira

Dutta, M. K. \& Modak, B., Can Noether symmetry in modified $f(G)$ gravity always yield cosmic evolution?
32 (2017) 1750170
32 (2017) 1750048
32 (2017) 1750139
32 (2017) 1750048
32 (2017) 1750139
32 (2017) 1750054
32 (2017) 1750139
32 (2017) 1750133
32 (2017) 1750170

32 (2017) 1750216

32 (2017) 1750139

32 (2017) 1750139

32 (2017) 1750144

32 (2017) 1750139

32 (2017) 1750139

32 (2017) 1750139

32 (2017) 1740007

32 (2017) 1750135

32 (2017) 1750220

32 (2017) 1750139

32 (2017) 1750139

32 1792002(E)

32 (2017) 1750100

32 (2017) 1750122

32 (2017) 1750139

32 (2017) 1750043

32 (2017) 1750031

32 (2017) 1750139

32 (2017) 1740013

32 (2017) 1750036

32 (2017) 1750139

32 (2017) 1750139

32 (2017) 1750108

32 (2017) 1750139

32 (2017) 1750139

32 (2017) 1730016

32 (2017) 1750046
Ebina, K., see Aaltonen

Egger, L., Maas, A. \& Sondenheimer, R., Pair production processes and flavor in gauge-invariant perturbation theory

Elagin, A., see Aaltonen

Eppig, A., see Aaltonen

Erbacher, R., see Aaltonen

Ernazarov, K. K., On non-exponential cosmological solutions with two factor spaces of dimensions $m$ and 1 in the Einstein-Gauss-Bonnet model with a $\Lambda$-term

Errede, D., see Aaltonen

Errede, S., see Aaltonen

Ershaidat, N., see Aaltonen

Escamilla-Rivera, C., see Castellanos

Espinoza, A., see Chubykalo

Esposito, J., see Maggiore

Eusebi, R., see Aaltonen

Fabbri, L., Singularity-free spinors in gravity with propagating torsion

Fan, W.-K., see Fan

Fan, Z.-Y., Fan, W.-K., Wang, Q.-W. \& Zong, H.-S., NJL model with the modified quark-dependent coupling strength $G$

Fang, H. C., see Aaltonen

Fariborz, A. H. \& Jora, R., Examining a possible cascade effect in chiral symmetry breaking

Farrington, S., see Aaltonen

Faustov, R. N. \& Galkin, V. O., Rare $\Lambda_{b} \rightarrow n l^{+} l^{-}$decays in the relativistic quark-diquark picture

Feindt, M., see Aaltonen

Feng, W.-Z. \& Nath, P., Baryogenesis and dark matter in $U(1)$ extensions

Fernandes, R. L., see Abreu

Fernandez, J. P., see Aaltonen

Fernando, S., see Balart

Fernando, S., Charged dilaton black hole in $2+1$ dimensions as a particle accelerator

Fernando, S., Spinning dilaton black hole in $2+1$ dimensions as a particle accelerator

Ferrazza, C., see Aaltonen

Field, R., see Aaltonen

Fischer, O., Clues on the Majorana scale from scalar resonances at the LHC

Fiziev, P. P., Compact static stars in minimal dilatonic gravity

Flambaum, V. V., see Stadnik
32 (2017) 1750139

32 (2017) 1750212

32 (2017) 1750139

32 (2017) 1750139

32 (2017) 1750139

32 (2017) 1750202

32 (2017) 1750139

32 (2017) 1750139

32 (2017) 1750139

32 (2017) 1750007

32 (2017) 1730033

32 (2017) 1740010

32 (2017) 1750139

32 (2017) 1750221

32 (2017) 1750107

32 (2017) 1750107

32 (2017) 1750139

32 (2017) 1750008

32 (2017) 1750139

32 (2017) 1750125

32 (2017) 1750139

32 (2017) 1740005

32 (2017) 1750019

32 (2017) 1750139

32 (2017) 1750219

32 (2017) 1750088

32 (2017) 1750074

32 (2017) 1750139

32 (2017) 1750139

32 (2017) 1750035

32 (2017) 1750141 32 (2017) 1740004 
Flanagan, G., see Aaltonen

Fomin, I. V. \& Chervon, S. V., A new approach to exact solutions construction in scalar cosmology with a Gauss-Bonnet term

Fornal, B., Dark matter and baryogenesis from non-Abelian gauged lepton number

Forrest, R., see Aaltonen

Forte, M., Kinematic equivalence between models driven by DBI field with constant $\gamma$ and exotic holographic quintessence cosmological models

Frampton, P. H., Cyclic period in the CBE model

Frampton, P. H., Second octant favored for non-maximal $\theta_{23}$

Frampton, P. H., Theory of dark matter

Franceschini, R., Energy peaks: A high energy physics outlook

Frank, M. J., see Aaltonen

Franklin, M., see Aaltonen

Freeman, J. C., see Aaltonen

Freitas, L. F. F., see Bezerra

Frenkel, J., see Britto

Fried, H. M., Grandou, T. \& Hofmann, R., On the non-perturbative realization of QCD gaugeinvariance

Fritzsch, H., Dark matter and excited weak bosons

Fritzsch, H., Excited weak bosons and their decays

Fujikawa, K. \& Oh, C. H., Exact algebraic separability criterion for two-qubit systems

Fukuyama, T., Addenda to general spin precession and betatron oscillation in storage ring

Fülöp, T., see Matolcsi

Funakoshi, Y., see Aaltonen

Furic, I., see Aaltonen

Galea, R., see Matei

Galkin, V. O., see Faustov

Gallinaro, M., see Aaltonen

Galyardt, J., see Aaltonen

Gamboa, J., see Arias

Gamboa, J., see Das

Ganai, P. A., Mir, M. A., Rafiqi, I. \& Ul Islam, N., Chern-Simons theory in aether superspace

Gangopadhyay, S., see Ghosh

Gangopadhyay, S., see Halder

Gangopadhyay, S., see Saha
32 (2017) 1750139

Gao, T.-J., Xiu, W.-T. \& Yang, X.-Y., Inflection point in running kinetic term inflation

Gao, Y., see Zhang

32 (2017) 1750129

32 (2017) 1730018

32 (2017) 1750139

Garcia, J. E., see Aaltonen

Garfinkel, A. F., see Aaltonen

Garosi, P., see Aaltonen

Gelbart, W., see Matei

Genish, A. \& Gepner, D., Generalized Rogers-Ramanujan identities for twisted affine algebras

Gepner, D., see Genish

Gerberich, H., see Aaltonen

32 (2017) 1750140

Gerbershagen, A., Hrbacek, J., Ijpes, D. \& Schippers, J. M., Transmis-

32 (2017) 1750132 sion improvement options via local energy degradation at a cyclotron driven ocular tumor treatment facility

32 (2017) 1750101

32 (2017) 1730013

32 (2017) 1730034

32 (2017) 1750139

32 (2017) 1750139

32 (2017) 1750139

32 (2017) 1750005

32 (2017) 1750171

Gerchtein, E., see Aaltonen

Ghilencea, D. M. \& Lee, H. M., Bounds on supersymmetric effective operators from heavy diphoton searches

Ghosh, M., Goswami, S. \& Raut, S. K., Implications of $\delta_{\mathrm{CP}}=-90^{\circ}$ towards determining hierarchy and octant at $\mathrm{T} 2 \mathrm{~K}$ and T2K-II

32 (2017) 1730030

32 (2017) 1730009

32 (2017) 1750057

32 (2017) 1750070

32 (2017) 1791001

32 (2017) 1750147

32 (2017) 1750139

32 (2017) 1750139

32 (2017) 1740011

32 (2017) 1750125

32 (2017) 1750139

32 (2017) 1750139

32 (2017) 1750032

32 (2017) 1750175

32 (2017) 1750214

32 (2017) 1750089

32 (2017) 1750102

32 (2017) 1750109
Ghosh, S. \& Gangopadhyay, S., Thermodynamics and emergent universe

Ghosh, S., see Das

Ghosh, S., see Saha

Ghosh, S., see Saha

Giagu, S., see Aaltonen

Giakoumopoulou, V., see Aaltonen

Giannetti, P., see Aaltonen

Gibson, K., see Aaltonen

Giné, J., see McCulloch

Ginsburg, C. M., see Aaltonen

Giokaris, N., see Aaltonen

Giovannini, M., Quantum coherence of cosmological perturbations

Giromini, P., see Aaltonen

Giunta, M., see Aaltonen

Giurgiu, G., see Aaltonen

Glagolev, V., see Aaltonen

Glenzinski, D., see Aaltonen

Gnatenko, Kh. P., Kinematic variables in noncommutative phase space and parameters of noncommutativity

Godinho, C. F. L., see Abreu

Golchehre, S. G., see Bakhshi

Gold, M., see Aaltonen
32 (2017) 1750072

32 (2017) 1750195

32 (2017) 1750139

32 (2017) 1750139

32 (2017) 1750139

32 (2017) 1740011

32 (2017) 1750110

32 (2017) 1750110

32 (2017) 1750139

32 (2017) 1740016

32 (2017) 1750139

32 (2017) 1750082

32 (2017) 1750034

32 (2017) 1750089

32 (2017) 1750180

32 (2017) 1750018

32 (2017) 1750109

32 (2017) 1750139

32 (2017) 1750139

32 (2017) 1750139

32 (2017) 1750139

32 (2017) 1750148

32 (2017) 1750139

32 (2017) 1750139

32 (2017) 1750191

32 (2017) 1750139

32 (2017) 1750139

32 (2017) 1750139

32 (2017) 1750139

32 (2017) 1750139

32 (2017) 1750166

32 (2017) 1750215

32 (2017) 1750161

32 (2017) 1750139 
Goldin, D., see Aaltonen Goldschmidt, N., see Aaltonen Golossanov, A., see Aaltonen Gomez, G., see Aaltonen Gomez-Ceballos, G., see Aaltonen Gómez-Valent, A., see Solà Goncharov, M., see Aaltonen González, O., see Aaltonen González, P. A., Olivares, M. \& Vásquez, Y., Bounded orbits for photons as a consequence of extra dimensions

Gonzalez-Sanchez, A., see Chubykalo

Gonzalez Suarez, R., Recent CMS results in top and Higgs physics

Gorelov, I., see Aaltonen

Goshaw, A. T., see Aaltonen

Goswami, R., see Maharaj

Goswami, S., see Ghosh

Goulianos, K., see Aaltonen

Grandou, T., see Fried

Gray, F., Santiago, J., Schuster, S. \& Visser, M., "Twisted" black holes are unphysical

Greiner, W., see Mişicu

Gresele, A., see Aaltonen

Grinstein, S., see Aaltonen

Gross, C., Lebedev, O. \& No, J. M., Drell-Yan constraints on new electroweak states: LHC as a $p p \rightarrow \ell^{+} \ell^{-}$precision machine

Grosso-Pilcher, C., see Aaltonen

Group, R. C., see Aaltonen

Guadagnoli, D., Flavor anomalies on the eve of the Run-2 verdict

Guan, R.-H., see Wang

Guendelman, E. I., see Vulfs

Guimaraes da Costa, J., see Aaltonen

Gunay-Unalan, Z., see Aaltonen

Gupta, K. S., see Sen

Gupta, V., see Polanco-Euán

Gusin, P. \& Radosz, A., The volume of the black holes - The constant curvature slicing of the spherically symmetric spacetime

Gutiérrez Rodríguez, A., see Chubykalo

Ha, Y. K., Weighing the black hole via quasi-local energy

Haber, C., see Aaltonen

Hahn, S. R., see Aaltonen

Haldar, P. K., see Mali

Halder, A. \& Gangopadhyay, S., Phase-space noncommutativity
32 (2017) 1750139 32 (2017) 1750139 32 (2017) 1750139 32 (2017) 1750139 32 (2017) 1750139 32 (2017) 1750054 32 (2017) 1750139 32 (2017) 1750139

32 (2017) 1750173

32 (2017) 1730033

32 (2017) 1730026

32 (2017) 1750139

32 (2017) 1750139

32 (2017) 1750164

32 (2017) 1750034

32 (2017) 1750139

32 (2017) 1730030

32 (2017) 1771001

32 (2017) 1750010

32 (2017) 1750139

32 (2017) 1750139

32 (2017) 1750094

32 (2017) 1750139

32 (2017) 1750139

32 (2017) 1730006

32 (2017) 1750044

32 (2017) 1750211

32 (2017) 1750139

32 (2017) 1750139

32 (2017) 1750217

32 (2017) 1750041

32 (2017) 1750115

32 (2017) 1730033

32 (2017) 1730021

32 (2017) 1750139

32 (2017) 1750139

32 (2017) 1750024 and the thermodynamics of the Landau system

Halilsoy, M., see Mazharimousavi

Halkiadakis, E., see Aaltonen

Halzen, F., IceCube in the era of multimessenger astrophysics

Hamaguchi, A., see Aaltonen

Hamil, B., Dirac oscillator in a space with spin noncommutativity of coordinates

Hammou, A. B., see Naceri

Han, J. Y., see Aaltonen

Hanif, S., see Bhatti

Hansraj, S. \& Qwabe, N., Inverse square law isothermal property in relativistic charged static distributions

Happacher, F., see Aaltonen

Hara, K., see Aaltonen

Hare, D., see Aaltonen

Hare, M., see Aaltonen

Harr, R. F., see Aaltonen

Hasegawa, C. \& Nakayama, Yu, $\epsilon$ expansion in critical $\phi^{3}$-theory on real projective space from conformal field theory

Hassanabadi, H., Alimohammadi, M. $\&$ Zare, S., $\gamma$-rigid version of Bohr Hamiltonian with the modified Davidson potential in the positiondependent mass formalism

Hassanabadi, H., see Chung

Hata, M., see Kawamura

Hatakeyama, K., see Aaltonen

Hays, C., see Aaltonen

He, Q. H., Study of event mixing for Bose-Einstein correlations in reactions with $\pi \pi X$ final states around $1 \mathrm{GeV}$

He, R., Qian, J., Chen, J., Wu, Q. \& Huo, L., Extracting temperature and transverse flow by fitting transverse mass spectra and HBT radii together

Heck, M., see Aaltonen

Heinrich, J., see Aaltonen

Herdy, W., see Accioly

Herdy, W., see Accioly

Hernandez, L. A., see Bisschoff

Hewamanage, S., see Aaltonen

Hidas, D., see Aaltonen

Hirayama, Y., see Kawamura

Hocker, A., see Aaltonen

Hoff da Silva, J. M., see Pereira

Hoff da Silva, J. M. \& Caval-
32 (2017) 1750102

32 (2017) 1750064

32 (2017) 1750139

32 (2017) 1730010

32 (2017) 1750139

32 (2017) 1750176

32 (2017) 1750120

32 (2017) 1750139

32 (2017) 1750042

32 (2017) 1750204

32 (2017) 1750139

32 (2017) 1750139

32 (2017) 1750139

32 (2017) 1750139

32 (2017) 1750139

32 (2017) 1750045

32 (2017) 1750085

32 (2017) 1750138

32 (2017) 1750058

32 (2017) 1750139

32 (2017) 1750139

32 (2017) 1750118

32 (2017) 1750052

32 (2017) 1750139

32 (2017) 1750139

32 (2017) 1750048

32 (2017) 1750185

32 (2017) 1750036

32 (2017) 1750139

32 (2017) 1750139

32 (2017) 1750058

32 (2017) 1750139

32 (2017) 1730016 
canti, R. T., Revealing how different spinors can be: The Lounesto spinor classification

Hofmann, R., see Fried

Hogan, P. A., see Böhmer

Hopkins, W., see Aaltonen

Horn, D., see Aaltonen

Hossein, Sk M., see Kalam

Hou, S., see Aaltonen

Hrbacek, J., see Gerbershagen

Hsu, L.-Y., Kawamoto, S. \& Wen, W.-Y., Entropic uncertainty relation based on generalized uncertainty principle

Huang, M. R., see Zhang

Huang, Y.-F., see Shu

Huang, Y.-F., see Shu

Hughes, R. E., see Aaltonen

Huo, L., see $\mathrm{He}$

Hurwitz, M., see Aaltonen

Husemann, U., see Aaltonen

Hussain, N., see Aaltonen

Hussain, T., see Khan

Hussain, T. \& Rahim, W., Homothetic matter collineations of LRS Bianchi type I spacetimes

Hussein, M., see Aaltonen

Huston, J., see Aaltonen

Hwang, J. K., New fermionic dark matters, extended Standard Model and cosmic rays

Ibort, A., see Asorey

Ibort, A., see Asorey

Ibort, A., see Asorey

Iizuka, J. \& Kitabayashi, T., Relativistic effective degrees of freedom and quantum statistics of neutrinos

Ijpes, D., see Gerbershagen

Ikeda, M., see Kawamura

Ikeda, Y., see Kawamura

Ilyas, M., see Yousaf

Infantino, A., see Cicoria

Introzzi, G., see Aaltonen

Iori, M., see Aaltonen

Ishii, T., see Kawamura

Islam, R., see Kalam

Ivanov, A., see Aaltonen

Jakovác, A., Kaposvári, I. \& Patkós, A., Scalar mass stability bound in a simple Yukawa-theory from renormalization group equations

Jakubowski, A., see Szkliniarz

James, E., see Aaltonen

Jang, D., see Aaltonen
32 (2017) 1730032

32 (2017) 1730030

32 (2017) 1750189

32 (2017) 1750139

32 (2017) 1750139

32 (2017) 1750012

32 (2017) 1750139

32 (2017) 1740016

32 (2017) 1750145

32 (2017) 1750195

32 (2017) 1750027

32 (2017) 1750209

32 (2017) 1750139

32 (2017) 1750052

32 (2017) 1750139

32 (2017) 1750139

32 (2017) 1750139

32 (2017) 1750124

32 (2017) 1750197

32 (2017) 1750139

32 (2017) 1750139

32 (2017) 1730023

32 1792002(E)

32 (2017) 1750100

32 (2017) 1750122

32 (2017) 1750069

32 (2017) 1740016

32 (2017) 1750058

32 (2017) 1750058

32 (2017) 1750163

32 (2017) 1740014

32 (2017) 1750139

32 (2017) 1750139

32 (2017) 1750058

32 (2017) 1750012

32 (2017) 1750139

32 (2017) 1750011

32 (2017) 1740012

32 (2017) 1750139

32 (2017) 1750139
Jarwal, B. \& Singh, S. S., Oscillation of boson star in Newtonian approximation

Jastrzębski, J., see Szkliniarz

Jayatilaka, B., see Aaltonen

Jenkovszky, L. \& Szanyi, I., Structures in the diffraction cone: The "break" and "dip" in high-energy proton-proton scattering

Jeon, E. J., see Aaltonen

Jha, M. K., see Aaltonen

Jiang, Y., see Li

Jindariani, S., see Aaltonen

Jing, Y.-L., see Xi

Johnson, R. R., see Matei

Johnson, W., see Aaltonen

Jones, M., see Aaltonen

Joo, K. K., see Aaltonen

Jora, R., see Fariborz

Jun, S. Y., see Aaltonen

Junk, T. R., see Aaltonen

Jusufi, K. \& Övgün, A., Canonical acoustic thin-shell wormholes

Kalam, M., Hossein, Sk M., Islam, R. \& Molla, S., Relativistic model of neutron stars in X-ray binary

Kameda, D., see Kawamura

Kamon, T., see Aaltonen

Kapinos, K., see Szkliniarz

Kaposvári, I., see Jakovác

Karchin, P. E., see Aaltonen

Kato, Y., see Aaltonen

Kaufman, J., see Leon

Kaur, K. \& Kumar, S., On the rapidity distribution of nucleons participating in elliptical flow at intermediate energies

Kawamoto, S., see Hsu

Kawamura, H., Akiyama, T., Hata, M., Hirayama, Y., Ikeda, M., Ikeda, Y., Ishii, T., Kameda, D., Mitsuoka, S., Miyatake, H., Nagae, D., Nakaya, Y., Ninomiya, K., Nitta, M., Ogawa, N., Onishi, J., Seitaibashi, E., Tanaka, S., Tanuma, R., Totsuka, Y., Toyoda, T., Watanabe, Y. X. \& Murata, J., A new measurement of electron transverse polarization in polarized nuclear $\beta$-decay

Kazantsev, A. E., Skoptsov, M. B. \& Stepanyantz, K. V., One-loop polarization operator of the quantum gauge superfield for $\mathcal{N}=1 \mathrm{SYM}$ regularized by higher derivatives
32 (2017) 1750037

32 (2017) 1740012

32 (2017) 1750139

32 (2017) 1750116

32 (2017) 1750139

32 (2017) 1750139

32 (2017) 1750013

32 (2017) 1750139

32 (2017) 1750160

32 (2017) 1740011

32 (2017) 1750139

32 (2017) 1750139

32 (2017) 1750139

32 (2017) 1750008

32 (2017) 1750139

32 (2017) 1750139

32 (2017) 1750047

32 (2017) 1750012

32 (2017) 1750058

32 (2017) 1750139

32 (2017) 1740012

32 (2017) 1750011

32 (2017) 1750139

32 (2017) 1750139

32 (2017) 1730002

32 (2017) 1750001 32 (2017) 1750145

32 (2017) 1750058

32 (2017) 1750194 
Keating, B., see Leon

Kepuladze, Z., see Chkareuli

Keskin, A. İ. \& Acikgoz, I., A unified picture of cosmological entropy on apparent horizon in $F(R, G)$ gravity

Ketchum, W., see Aaltonen

Keung, J., see Aaltonen

Khalilov, V. R., Quasi-stationary states and fermion pair creation from a vacuum in supercritical Coulomb field

Khan, F., Hussain, T. \& Akhtar, S. S., Conformal Ricci collineations in LRS Bianchi type V spacetimes with perfect fluid matter

Khan, S., Shah, H., Ahmad, Z. \& Ramzan, M., Final fate of charged anisotropic fluid collapse

Khatsymovsky, V. M., First-order discrete Faddeev gravity at strongly varying fields

Khlopov, M. Yu., Introduction

Khlopov, M. Yu., Dark matter reflection of particle symmetry

Khlopov, M. Yu., see Addazi

Khodaee, S. \& Vassilevich, D., Note on correlation functions in conformal quantum mechanics

Kholodov, R. I., see Diachenko

Khoo, K. S., see Salih

Khotilovich, V., see Aaltonen

Khurshudyan, As. Zh., see Avetisyan

Khurshudyan, As., see Khurshudyan Khurshudyan, As., see Khurshudyan Khurshudyan, M. \& Khurshudyan, As., Phenomenological modification of horizon temperature

Khurshudyan, M., Myrzakulov, R. \& Khurshudyan, As., Interacting dark energy models in $f(T)$ gravity

Kilminster, B., see Aaltonen

Kim, D. H., see Aaltonen

Kim, H. S., see Aaltonen

Kim, H. W., see Aaltonen

Kim, J. E., see Aaltonen

Kim, J., Son, H.-J. \& Park, Y.-H., Design for simultaneous acceleration of stable and unstable beams in a superconducting heavy-ion linear accelerator for RISP

Kim, M. J., see Aaltonen

Kim, S. B., see Aaltonen
32 (2017) 1730002 32 (2017) 1750029

32 (2017) 1750182
32 (2017) 1750139
32(2017) 1750139

32 (2017) 1750200

32 (2017) 1750124

32 (2017) 1750192

32 (2017) 1750181

32 (2017) 1702001

32 (2017) 1740001

32 (2017) 1740002

32 (2017) 1750157

32 (2017) 1750031

32 (2017) 1750065

32 (2017) 1750139

32 (2017) 1730015

32 (2017) 1750097

32 (2017) 1750156

32 (2017) 1750156

32 (2017) 1750097

32 (2017) 1750139

32 (2017) 1750139

32 (2017) 1750139

32 (2017) 1750139

32 (2017) 1750139

32 (2017) 1750203

32 (2017) 1750139

32 (2017) 1750139
Kim, S. H., see Aaltonen

Kim, Y. K., see Aaltonen

Kimura, N., see Aaltonen

Kirby, M., see Aaltonen

Kirilova, D. P. \& Chizhov, V. M., Chiral tensor particles in the early Universe - Present status

Kirschner, R. \& Savvidy, G., Yangian and SUSY symmetry of high spin parton splitting amplitudes in generalised Yang-Mills theory

Kisieliński, M., see Szkliniarz

Kitabayashi, T., see Iizuka

Kitazawa, N., On D-brane dynamics and moduli stabilization

Klimenko, S., see Aaltonen

Klinkhamer, F. R. \& Volovik, G. E., Propagating $q$-field and $q$-ball solution

Kobakhidze, A., see Barrie

Kobayashi, T., Seto, O., Shimomura, T. \& Urakawa, Y., Relaxion window

Koide, Y., Sumino's cancellation mechanism in an anomaly-free model

Kondo, K., see Aaltonen

Kong, D. J., see Aaltonen

Konigsberg, J., see Aaltonen

Korenblit, S. E. \& Sinitskaya, A. V., On asymptotic power corrections to differential fluxes and generalization of optical theorem for potential scattering

Kota, V. K. B., see Sahu

Kotwal, A. V., see Aaltonen

Kováčik, S., $R_{\lambda}^{3}$-inspired black holes

Kovtun, A. V. \& Nugaev, E. Ya., Radiative corrections and instability of large Q-balls

Koyuncu, F., Soylu, A. \& Bayrak, O., Binary cluster model calculations for ${ }^{20} \mathrm{Ne}$ and ${ }^{44} \mathrm{Ti}$ nuclei

Kremer, G. M., see Ribas

Kremer, G. M., see Ribas

Kreps, M., see Aaltonen

Krishnan, C. \& Raju, A., A Neumann boundary term for gravity

Kroll, J., see Aaltonen

Krop, D., see Aaltonen

Kruglov, S. I., Remarks on Heisenberg-Euler-type electrodynamics

Kruglov, S. I., Notes on Born-Infeldtype electrodynamics

Krumnack, N., see Aaltonen
32 (2017) 1750139

32 (2017) 1750139

32 (2017) 1750139

32 (2017) 1750139

32 (2017) 1750187

32 (2017) 1750121

32 (2017) 1740012

32 (2017) 1750069

32 (2017) 1750150

32 (2017) 1750139

32 (2017) 1750103

32 (2017) 1750087

32 (2017) 1750142

32 (2017) 1750062

32 (2017) 1750139

32 (2017) 1750139

32 (2017) 1750139

32 (2017) 1750066

32 (2017) 1750210

32 (2017) 1750139

32 (2017) 1750130

32 (2017) 1750198

32 (2017) 1750050

32 (2017) 1750135

32 (2017) 1750220

32 (2017) 1750139

32 (2017) 1750077

32 (2017) 1750139

32 (2017) 1750139

32 (2017) 1750092

32 (2017) 1750201

32 (2017) 1750139 
Kruse, M., see Aaltonen

Krutelyov, V., see Aaltonen

Kuhr, T., see Aaltonen

Kumar, R., Singh, P. \& Kumar, R., Analysis of longitudinal momentum distribution data of ${ }^{26-29} \mathrm{P}$ isotopes in stripping reactions

Kumar, R., see Kumar

Kumar, S., see Kaur

Kumar, S., see Channey

Kurata, M., see Aaltonen

Kwang, S., see Aaltonen

Laasanen, A. T., see Aaltonen

Lai, J.-T. \& Yue, C.-X., Light gauge boson $Z^{\prime}$ and LFV decays of the electroweak gauge boson $Z$

Lami, S., see Aaltonen

Lammel, S., see Aaltonen

Lancaster, M., see Aaltonen

Lander, R. L., see Aaltonen

Landim, R. R., Tahim, M. O., Alencar, G. \& Costa Filho, R. N., Analytical solutions for fermions on a thick brane with a piecewise and smooth warp factor

Langille, S., see Matei

Lannon, K., see Aaltonen

Lath, A., see Aaltonen

Latino, G., see Aaltonen

Lavrov, P. M., see Batalin

Lazzizzera, I., see Aaltonen

Lebedev, O., see Gross

LeCompte, T., see Aaltonen

Lee, C. H., see Salih

Lee, E., see Aaltonen

Lee, H. M., see Ghilencea

Lee, H. S., see Aaltonen

Lee, J. S., see Aaltonen

Lee, S. W., see Aaltonen

Leo, S., see Aaltonen

Leon, D., Kaufman, J., Keating, B. \& Mewes, M., The cosmic microwave background and pseudoNambu-Goldstone bosons: Searching for Lorentz violations in the cosmos

Leone, S., see Aaltonen

Leonte, R., see Matei

Lev, B. I., see Lev

Lev, S. B. \& Lev, B. I., Nonequilibrium dynamic of a Universe formation

Li, C.-M., see Zhao

Li, H.-F., Ma, M.-S. \& Ma, Y.-Q., Thermodynamic properties of black holes in de Sitter space
32 (2017) 1750139

32 (2017) 1750139

32 (2017) 1750139

32 (2017) 1750021

32 (2017) 1750021

32 (2017) 1750001

32 (2017) 1750137

32 (2017) 1750139

32 (2017) 1750139

32 (2017) 1750139

32 (2017) 1750039

32 (2017) 1750139

32 (2017) 1750139

32 (2017) 1750139

32 (2017) 1750139

32 (2017) 1750193

32 (2017) 1740011

32 (2017) 1750139

32 (2017) 1750139

32 (2017) 1750139

32 (2017) 1750068

32 (2017) 1750139

32 (2017) 1750094

32 (2017) 1750139

32 (2017) 1750065

32 (2017) 1750139

32 (2017) 1750082

32 (2017) 1750139

32 (2017) 1750139

32 (2017) 1750139

32 (2017) 1750139

32 (2017) 1730002

32 (2017) 1750139

32 (2017) 1740011

32 (2017) 1750165

32 (2017) 1750165

32 (2017) 1750051

32 (2017) 1750017
Li, Q., see Li

Li, S.-C., Jiang, Y., Wang, T.-H., Li, Q., Wang, Z.-H. \& Wang, G.-L., Semi-leptonic production of $D_{s j}(3040)$ and $D_{j}(3000)$ in $B_{s}$ and $B$ decays

Likhoded, A. K., see Belyaev

Lin, C.-J., see Aaltonen

Lin, K. \& Wu, Y., Holographic superconductors in Einstein-æther gravity

Lin, K., Qian, W.-L., Pavan, A. B. \& Abdalla, E., A matrix method for quasinormal modes: Kerr and Kerr-Sen black holes

Linacre, J., see Aaltonen

Lindgren, M., see Aaltonen

Lipeles, E., see Aaltonen

Lister, A., see Aaltonen

Litvintsev, D. O., see Aaltonen

Liu, C., see Aaltonen

Liu, H. \& Meng, X.-H., Thermodynamics of Schwarzschild-Beltrami-de Sitter black hole

Liu, M. X., Prospects of direct search for dark photon and dark Higgs in SeaQuest/E1067 experiment at the Fermilab main injector

Liu, Q., see Aaltonen

Liu, T., see Aaltonen

Liu, X., see Zhang

Lockwitz, S., see Aaltonen

Lockyer, N. S., see Aaltonen

Loginov, A., see Aaltonen

Lombardi, A., see Maggiore

Lü, W.-D., see Xi

Lucchesi, D., see Aaltonen

Luchinsky, A. V., see Belyaev

Ludwick, K. J., The viability of phantom dark energy: A review

Lueck, J., see Aaltonen

Lujan, P., see Aaltonen

Lungu, G., see Aaltonen

Luo, M. J., Quark-gluon plasma and topological quantum field theory

Luo, X.-L., see Zhao

Lys, J., see Aaltonen

Lysak, R., see Aaltonen

Ma, B.-Q., see Yu

Ma, E., The many guises of a neutral fermion singlet

Ma, E., Self-interacting dark matter with naturally light mediator

Ma, M.-S., see Li

Ma, Y.-Q., see Li

Maas, A., see Egger
32 (2017) 1750013

32 (2017) 1750013

32 (2017) 1771002

32 (2017) 1750139

32 (2017) 1750188

32 (2017) 1750134

32 (2017) 1750139

32 (2017) 1750139

32 (2017) 1750139

32 (2017) 1750139

32 (2017) 1750139

32 (2017) 1750139

32 (2017) 1750146

32 (2017) 1730008

32 (2017) 1750139

32 (2017) 1750139

32 (2017) 1750195

32 (2017) 1750139

32 (2017) 1750139

32 (2017) 1750139

32 (2017) 1740010

32 (2017) 1750160

32 (2017) 1750139

32 (2017) 1771002

32 (2017) 1730025

32 (2017) 1750139

32 (2017) 1750139

32 (2017) 1750139

32 (2017) 1750056

32 (2017) 1750051

32 (2017) 1750139

32 (2017) 1750139

32 (2017) 1750030

32 (2017) 1730007

32 (2017) 1750038

32 (2017) 1750017

32 (2017) 1750017

32 (2017) 1750212 
Madrak, R., see Aaltonen

Maeshima, K., see Aaltonen

Maggiore, M., Campo, D., Antonini, P., Lombardi, A., Manzolaro, M., Andrighetto, A., Monetti, A., Scarpa, D., Esposito, J. \& Silvestrin, L., SPES: A new cyclotronbased facility for research and applications with high-intensity beams

Maharaj, S. D., see Rahaman

Maharaj, S. D., Goswami, R., Chervon, S. V. \& Nikolaev, A. V., Exact solutions for scalar field cosmology in $f(R)$ gravity

Mahdavi, M., see Azadifar

Majhi, A., Energy spectrum of black holes: A new view

Majumdar, P., see Sinha

Makhoul, K., see Aaltonen

Maksimovic, P., see Aaltonen

Mali, P., Mukhopadhyay, A., Manna, S. K., Haldar, P. K. \& Singh, G., Multifractal analysis of charged particle distributions using horizontal visibility graph and sandbox algorithm

Malik, S., see Aaltonen

Mallick, A., see Chakraborty

Manca, G., see Aaltonen

Manna, S. K., see Mali

Manousakis-Katsikakis, A., see Aaltonen

Manzolaro, M., see Maggiore

Marachlian, E., Sánchez G., I. E. \& Santillán, O. P., Emergent universe as an interaction in the dark sector

Marengo, M., see Cicoria

Margaroli, F., see Aaltonen

Marino, C., see Aaltonen

Marmo, G., see Asorey

Martínez, M., see Aaltonen

Martínez-Ballarín, R., see Aaltonen

Mastrandrea, P., see Aaltonen

Matei, L., McRae, G., Galea, R., Niculae, D., Craciun, L., Leonte, R., Surette, G., Langille, S., St. Louis, C., Gelbart, W., Abeysekera, B. \& Johnson, R. R., A new approach for manufacturing and processing targets to produce ${ }^{99 m} \mathrm{Tc}$ with cyclotrons

Mathis, M., see Aaltonen

Matolcsi, T., Fülöp, T. \& Weiner, M., Second-order equation of mo-
32 (2017) 1750139 32 (2017) 1750139

32 (2017) 1740010

32 (2017) 1750053

32 (2017) 1750164

32 (2017) 1750016

32 (2017) 1750002

32 (2017) 1750208

32 (2017) 1750139

32 (2017) 1750139

(2017) 1750024

32 (2017) 1750139

32 (2017) 1750055

32 (2017) 1750139

32 (2017) 1750024

32 (2017) 1750139

32 (2017) 1740010

32 (2017) 1750152

32 (2017) 1740014

32 (2017) 1750139

32 (2017) 1750139

32 (2017) 1750122

32 (2017) 1750139

32 (2017) 1750139

32 (2017) 1750139

32 (2017) 1740011 32 (2017) 1750139

1

tion for electromagnetic radiation back-reaction

Mattson, M. E., see Aaltonen

Matveev, M. A., see Anisovich

Mazharimousavi, S. H., Amirabi, Z. \& Halilsoy, M., Thin-shell wormholes in $(2+1)$-dimensional Einstein-scalar theory

Mazzanti, P., see Aaltonen

McCulloch, M. E. \& Giné, J., Modified inertial mass from information loss

McFarland, K. S., see Aaltonen

McIntyre, P., see Aaltonen

McNulty, R., see Aaltonen

McRae, G., see Matei

Mebarki, N., see Bendjoudi

Mehta, A., see Aaltonen

Mehtala, P., see Aaltonen

Mendes, A. C. R., see Abreu

Méndez, F., see Arias

Méndez, F., see Das

Meng, X.-H., see Liu

Menzione, A., see Aaltonen

Merad, M., see Benchikha

Mesropian, C., see Aaltonen

Mewes, M., see Leon

Miao, T., see Aaltonen

Mietlicki, D., see Aaltonen

Mir, M. A., see Ganai

Mirjalili, A., see Taghavi

Mishustin, I. N., see Mişicu

Mişicu, Ş., Mishustin, I. N. \& Greiner, W., $Q$-balls of clusterized baryonic matter

Mitra, A., see Aaltonen

Mitsuoka, S., see Kawamura

Miyake, H., see Aaltonen

Miyatake, H., see Kawamura

Mizuk, R. V., see Bondar

Modak, B., see Biswas

Modak, B., see Dutta

Modak, T. \& Srivastava, R., Probing anomalous Higgs couplings in $H \rightarrow Z V$ decays

Moed, S., see Aaltonen

Moggi, N., see Aaltonen

Mohapatra, R. N., see Dev

Molinaro, E. \& Vignaroli, N., Diphoton resonances at the LHC

Molla, S., see Kalam

Momen, A., see Balachandran

Mondragon, M. N., see Aaltonen

Monetti, A., see Maggiore

Moon, C. S., see Aaltonen
32 (2017) 1750147

32 (2017) 1750139

32 (2017) 1750154

32 (2017) 1750064

32 (2017) 1750139

32 (2017) 1750148

32 (2017) 1750139

32 (2017) 1750139

32 (2017) 1750139

32 (2017) 1740011

32 (2017) 1750015

32 (2017) 1750139

32 (2017) 1750139

32 (2017) 1750019

32 (2017) 1750032

32 (2017) 1750175

32 (2017) 1750146

32 (2017) 1750139

32 (2017) 1750106

32 (2017) 1750139

32 (2017) 1730002

32 (2017) 1750139

32 (2017) 1750139

32 (2017) 1750214

32 (2017) 1750040

32 (2017) 1750010

32 (2017) 1750010

32 (2017) 1750139

32 (2017) 1750058

32 (2017) 1750139

32 (2017) 1750058

32 (2017) 1750025

32 (2017) 1750023

32 (2017) 1750046

32 (2017) 1750004

32 (2017) 1750139

32 (2017) 1750139

32 (2017) 1740007

32 (2017) 1730024

32 (2017) 1750012

32 (2017) 1750133

32 (2017) 1750139

32 (2017) 1740010

32 (2017) 1750139 
Moore, R., see Aaltonen

Moradpour, H., Nunes, R. C., Abreu, E. M. C. \& Neto, J. A., A note on the relations between thermodynamics, energy definitions and Friedmann equations

Morello, M. J., see Aaltonen

Morlock, J., see Aaltonen

Movilla Fernandez, P., see Aaltonen

Mukherjee, A., see Aaltonen

Mukherjee, T. K. \& Sanyal, S., Particle temperature and the chiral vortical effect in the early universe

Mukhopadhyay, A., see Mali

Muller, Th., see Aaltonen

Muniz, C. R., see Bezerra

Murat, P., see Aaltonen

Murata, J., see Kawamura

Mussini, M., see Aaltonen

Myrzakulov, R., see Khurshudyan

Naceri, L. \& Hammou, A. B., The analytical transfer matrix method for PT-symmetric complex potential

Nachtman, J., see Aaltonen

Nagae, D., see Kawamura

Nagai, Y., see Aaltonen

Naganoma, J., see Aaltonen

Nakano, I., see Aaltonen

Nakaya, Y., see Kawamura

Nakayama, Yu, see Hasegawa

Nam, S. i., Quasi-distribution amplitudes for pion and kaon via the nonlocal chiral-quark model

Nandan, H., see Tripathi

Nandi, B. K., see Dash

Napier, A., see Aaltonen

Nath, P., see Feng

Nawazish, I., see Sharif

Nayak, R., see Dash

Nazir, K., see Sharif

Nefedov, M. \& Saleev, V., On the one-loop calculations with Reggeized quarks

Neto, J. A., see Abreu

Neto, J. A., see Moradpour

Nett, J., see Aaltonen

$\mathrm{Neu}, \mathrm{C}$., see Aaltonen

Neubauer, M. S., see Aaltonen

Neves, A. C. B., see Alves

Neves, M. Jr., see Abreu

Ngo, Q. T. \& Shinmura, S., Constraints on the equation of state of neutron star matter based on observations
32 (2017) 1750139

32 (2017) 1750078
32 (2017) 1750139
32 (2017) 1750139
$\mathbf{3 2}(2017) 1750139$
32 (2017) 1750139

32 (2017) 1750178

32 (2017) 1750024

32 (2017) 1750139

32 (2017) 1750005

32 (2017) 1750139

32 (2017) 1750058

32 (2017) 1750139

32 (2017) 1750097

32 (2017) 1750120

32 (2017) 1750139

32 (2017) 1750058

32 (2017) 1750139

32 (2017) 1750139

32 (2017) 1750139

32 (2017) 1750058

32 (2017) 1750045

32 (2017) 1750218

32 (2017) 1750061

32 (2017) 1750060

32 (2017) 1750139

32 (2017) 1740005

32 (2017) 1750136

32 (2017) 1750060

32 (2017) 1750083

32 (2017) 1750207

32 (2017) 1750019

32 (2017) 1750078

32 (2017) 1750139

32 (2017) 1750139

32 (2017) 1750139

32 (2017) 1740013

32 (2017) 1750019

32 (2017) 1750190
Nguyen, A. V. \& Pham, T. D., NonAbelian gauge fields as components of gravity in the discretized Kaluza-Klein theory

Ni, G.-J. \& Cheung, I. P., Are there discrete symmetries in relativistic quantum mechanics?

Niculae, D., see Matei

Nielsen, J., see Aaltonen

Nikolaev, A. V., see Maharaj

Ninomiya, K., see Kawamura

Nitta, M., see Kawamura

No, J. M., see Gross

Nodulman, L., see Aaltonen

Nojiri, S., see Rotondo

Normann, B. D. \& Brevik, I., Characteristic properties of two different viscous cosmology models for the future universe

Norniella, O., see Aaltonen

Nugaev, E. Ya., see Kovtun

Nunes, R. C., see Moradpour

Nurse, E., see Aaltonen

Nyiri, J., see Anisovich

Oakes, L., see Aaltonen

Oda, I., Schwarzschild solution from Weyl transverse gravity

Odintsov, S. D., see Bamba

Ogawa, N., see Kawamura

Oh, C. H., see Fujikawa

Oh, C. H., see Zhang

Oh, S. H., see Aaltonen

Oh, Y. D., see Aaltonen

Oikonomou, V. K., On the expanding phase of a singular bounce and intermediate inflation: The modified gravity description

Oikonomou, V. K., Reheating in constant-roll $F(R)$ gravity

Oksuzian, I., see Aaltonen

Okusawa, T., see Aaltonen

Olivares, M., see González

Onishi, J., see Kawamura

Orava, R., see Aaltonen

Ortolan, L., see Aaltonen

Övgün, A., see Jusufi

Övgün, A. \& Salako, I. G., Thinshell wormholes in neo-Newtonian theory

Pagan Griso, S., see Aaltonen

Pagliarone, C., see Aaltonen

Palencia, E., see Aaltonen

Paliathanasis, A., Dust fluid component from Lie symmetries in scalar field cosmology
32 (2017) 1750095

32 (2017) 1730029

32 (2017) 1740011

32 (2017) 1750139

32 (2017) 1750164

32 (2017) 1750058

32 (2017) 1750058

32 (2017) 1750094

32 (2017) 1750139

32 (2017) 1750149

32 (2017) 1750026

32 (2017) 1750139

32 (2017) 1750198

32 (2017) 1750078

32 (2017) 1750139

32 (2017) 1750154

32 (2017) 1750139

32 (2017) 1750022

32 (2017) 1750114

32 (2017) 1750058

32 (2017) 1750070

32 (2017) 1750020

32 (2017) 1750139

32 (2017) 1750139

32 (2017) 1750067

32 (2017) 1750172

32 (2017) 1750139

32 (2017) 1750139

32 (2017) 1750173

32 (2017) 1750058

32 (2017) 1750139

32 (2017) 1750139

32 (2017) 1750047

32 (2017) 1750119

32 (2017) 1750139

32 (2017) 1750139

32 (2017) 1750139

32 (2017) 1750206 
Panahi, H., see Bakhshi

Pandey, A. K., see Dash

Pant, N., see Singh

Papadimitriou, V., see Aaltonen

Paramonov, A. A., see Aaltonen

Park, Y.-H., see Kim

Parkhomenko, S. E., Generalized

Kähler geometry and current algebras in $\mathrm{SU}(2) \times \mathrm{U}(1) N=2$ superconformal WZW model

Patkós, A., see Jakovác

Patrick, J., see Aaltonen

Paul, B. C., see Debnath

Pauletta, G., see Aaltonen

Paulini, M., see Aaltonen

Paus, C., see Aaltonen

Pavan, A. B., see Lin

Pellett, D. E., see Aaltonen

Peng, H., see Xie

Penzo, A., see Aaltonen

Pereira, S. H., Hoff da Silva, J. M. \& dos Santos, R., Casimir effect for Elko fields

Pham, T. D., see Nguyen

Phillips, T. J., see Aaltonen

Piacentino, G., see Aaltonen

Pianori, E., see Aaltonen

Pilot, J., see Aaltonen

Pincak, R., see Sepehri

Ping, J. L., see Wu

Plager, C., see Aaltonen

Polanco-Euán, E. N., Sánchez-Colón, G. \& Gupta, V., Dibaryon octet strong decay coupling constants sum rules with first-order SU(3) symmetry breaking

Pondrom, L., see Aaltonen

Potamianos, K., see Aaltonen

Poukhov, O., see Aaltonen

Pradhan, A., see Sepehri

Pradhan, P., Mass-independent area (or entropy) and thermodynamic volume products in conformal gravity

Prokoshin, F., see Aaltonen

Pronko, A., see Aaltonen

Ptohos, F., see Aaltonen

Pueschel, E., see Aaltonen

Punzi, G., see Aaltonen

Purohit, K. D., see Tripathi

Pursley, J., see Aaltonen

Qian, J., see He

Qian, W.-L., see Lin

Qiang, L.-E, see Bao

Qwabe, N., see Hansraj

$32(2017) 1750161$
$32(2017) 1750060$
$32(2017) 1750093$
$32(2017) 1750139$
$\mathbf{3 2}(2017) 1750139$
$\mathbf{3 2}(2017) 1750203$

Radiman, S., see Salih

Radosz, A., see Gusin

Rafiqi, I., see Ganai

Rahaman, A., see Aaltonen

Rahaman, F., Maharaj, S. D., Sardar,

I. H. \& Chakraborty, K., Conformally symmetric relativistic star

Rahaman, F., see Chakraborty

Rahaman, F., see Singh

32 (2017) 1750076

32 (2017) 1750011

32 (2017) 1750139

32 (2017) 1750216

32 (2017) 1750139

32 (2017) 1750139

32 (2017) 1750139

32 (2017) 1750134

32 (2017) 1750139

32 (2017) 1750081

32 (2017) 1750139

32 (2017) 1730016

32 (2017) 1750095

32 (2017) 1750139

32 (2017) 1750139

32 (2017) 1750139

32 (2017) 1750139

32 (2017) 1750033

32 (2017) 1750222

32 (2017) 1750139

32 (2017) 1750041

32 (2017) 1750139

32 (2017) 1750139

32 (2017) 1750139

32 (2017) 1750123

32 (2017) 1750090

32 (2017) 1750139

32 (2017) 1750139

32 (2017) 1750139

32 (2017) 1750139

32 (2017) 1750139

32 (2017) 1750061

32 (2017) 1750139

32 (2017) 1750052

32 (2017) 1750134

32 (2017) 1750131

32 (2017) 1750204
Rahaman, M., see Singh

Rahim, W., see Hussain

Raju, A., see Krishnan

Ramakrishnan, V., see Aaltonen

Ramzan, M., see Khan

Ranjan, N., see Aaltonen

Raut, S. K., see Ghosh

Redondo, I., see Aaltonen

Renton, P., see Aaltonen

Rescigno, M., see Aaltonen

Ribas, M. O., Devecchi, F. P. \& Kremer, G. M., Cosmology with fermionic sources and relativistic fluid in Schutz's formalism: Classical and quantum solutions

Ribas, M. O., Devecchi, F. P. \& Kremer, G. M., Fermionic and bosonic fields in the Einstein-Cartan theory

Ricciardi, G., Semileptonic and leptonic $B$ decays, circa 2016

Rifuggiato, D., see Calabretta

Riles, K., Recent searches for continuous gravitational waves

Rimondi, F., see Aaltonen

Ristori, L., see Aaltonen

Robson, A., see Aaltonen

Rodrigo, T., see Aaltonen

Rodriguez, T., see Aaltonen

Rogers, E., see Aaltonen

Rolli, S., see Aaltonen

Romero, R., On the nature of the neutrino

Roser, R., see Aaltonen

Rossi, M., see Aaltonen

Rotondo, M. \& Nojiri, S., A toy model of discretized gravity in two dimensions and its extensions

Rubbo, F., see Aaltonen

Rubin, S., see Belotsky

Ruffini, F., see Aaltonen

Ruiz, A., see Aaltonen

Russ, J., see Aaltonen

Russo, A. D., see Calabretta

Rusu, V., see Aaltonen

Rzehak, H., see Alanne
32 (2017) 1750065

32 (2017) 1750115

32 (2017) 1750214

32 (2017) 1750139

32 (2017) 1750053

32 (2017) 1750055

32 (2017) 1750093

32 (2017) 1750093

32 (2017) 1750197

32 (2017) 1750077

32 (2017) 1750139

32 (2017) 1750192

32 (2017) 1750139

32 (2017) 1750034

32 (2017) 1750139

32 (2017) 1750139

32 (2017) 1750139

32 (2017) 1750220

32 (2017) 1750135

32 (2017) 1730005

32 (2017) 1740009

32 (2017) 1730035

32 (2017) 1750139

32 (2017) 1750139

32 (2017) 1750139

32 (2017) 1750139

32 (2017) 1750139

32 (2017) 1750139

32 (2017) 1750139

32 1792001(E)

32 (2017) 1750139

32 (2017) 1750139

32 (2017) 1750149

32 (2017) 1750139

32 (2017) 1740008

32 (2017) 1750139

32 (2017) 1750139

32 (2017) 1750139

32 (2017) 1740009

32 (2017) 1750139

32 (2017) 1750143 
Sadiq, M., The holographic principle, the equipartition of energy and Newton's gravity

Sadiq, S., see Sharif

Safonov, A., see Aaltonen

Sagnotti, A., Low- $\ell$ CMB from string-scale SUSY breaking?

Saha, A., see Allen

Saha, K., Upadhaya, S. \& Ghosh, S., A comparative study on two different approaches of bulk viscosity in the Polyakov-Nambu-JonaLasinio model

Saha, S., Ghosh, S. \& Gangopadhyay, S., Interacting Chaplygin gas revisited

Şahin, B., Probing CP-violating $W W \gamma$ couplings in $\gamma p$ collision at the LHC

Sahoo, P. K., Sahoo, P., Bishi, B. K. \& Aygün, S., Magnetized strange quark model with Big Rip singularity in $f(R, T)$ gravity

Sahoo, P., see Sahoo

Sahu, R. \& Kota, V. K. B., Deformed shell model study of event rates for WIMP- ${ }^{73}$ Ge scattering

Sakumoto, W. K., see Aaltonen

Sakurai, Y., see Aaltonen

Salako, I. G., see Övgün

Saleem, U., see Sarfraz

Saleem, U., see Sarfraz

Saleev, V., see Nefedov

Salih, F. H. M., Lee, C. H., Radiman, S. \& Khoo, K. S., Nuclear structure study of two-neutron halo nucleus ${ }^{19} \mathrm{~B}$ in microscopic cluster model

Salti, M., Aydogdu, O., Yanar, H. \& Binbay, F., Brans-Dicke type teleparallel scalar-tensor theory

Salti, M., see Askin

Sammarruca, F., The symmetry energy: Predictions and constraints

Sampaio, M., see Alexander

Sánchez-Colón, G., see PolancoEuán

Sánchez G., I. E., see Marachlian

Sannino, F., see Alanne

Santi, L., see Aaltonen

Santiago, J., see Gray

Santillán, O. P., see Marachlian

Sanyal, S., see Mukherjee

Sardar, I. H., see Rahaman

Sarfraz, H. \& Saleem, U., Ex- act solutions of the noncommutative and commutative nonlinear Schrödinger equation in $2+1$ dimensions

32 (2017) 1750091

32 (2017) 1750139

32 (2017) 1730001

32 (2017) 1730022

Sarfraz, H. \& Saleem, U., Darboux transformation and multi-soliton solutions of local/nonlocal $N$-wave interactions

Saridakis, E. N., see Bamba

Sartori, L., see Aaltonen

Sato, K., see Aaltonen

Saveliev, V., see Aaltonen

32 (2017) 1750018

Savoy-Navarro, A., see Aaltonen

Savvidy, G., see Kirschner

Saw, V.-L., Mass loss due to gravitational waves with $\Lambda>0$

Scampoli, P., see Braccini

Scarpa, D., see Maggiore

32 (2017) 1750205

Schippers, J. M., see Gerbershagen

Schlabach, P., see Aaltonen

Schmidt, A., see Aaltonen

Schmidt, E. E., see Aaltonen

32 (2017) 1750105

32 (2017) 1750105

Schmidt, M. P., see Aaltonen

Schmitt, M., see Aaltonen

Schuster, S., see Gray

Schwartz, C., Revised theory of tachyons in general relativity

32 (2017) 1750210

32 (2017) 1750139

32 (2017) 1750139

32 (2017) 1750119

32 (2017) 1750158

32 (2017) 1750196

32 (2017) 1750207

Schwarz, T., see Aaltonen

Scodellaro, L., see Aaltonen

Scribano, A., see Aaltonen

Scuri, F., see Aaltonen

Sedov, A., see Aaltonen

Seidel, S., see Aaltonen

Seitaibashi, E., see Kawamura

Seiya, Y., see Aaltonen

Semenov, A., see Aaltonen

Semenova, A. N., see Anisovich

32 (2017) 1750065

32 (2017) 1750183

32 (2017) 1750177

32 (2017) 1730027

32 (2017) 1750104

32 (2017) 1750041

32 (2017) 1750152

32 (2017) 1750143

32 (2017) 1750139

32 (2017) 1771001

32 (2017) 1750152

32 (2017) 1750178

32 (2017) 1750053
Sen, S. \& Gupta, K. S., Observable consequences of zero-point energy

Senjanović, G., Is left-right symmetry the key?

Sepehri, A. \& Pincak, R., The birth of the universe in a new G-theory approach

Sepehri, A., Pradhan, A. \& Beesham, A., On the origin of generalized uncertainty principle from compactified $M 5$-brane

Seto, O., see Kobayashi

Sett, P., see Dash

Sforza, F., see Aaltonen

Sfyrla, A., see Aaltonen

Shah, H., see Khan

Shalhout, S. Z., see Aaltonen
32 (2017) 1750158

32 (2017) 1750196

32 (2017) 1750114

32 (2017) 1750139

32 (2017) 1750139

32 (2017) 1750139

32 (2017) 1750139

32 (2017) 1750121

32 (2017) 1730020

32 (2017) 1702002

32 (2017) 1740010

32 (2017) 1740016

32 (2017) 1750139

32 (2017) 1750139

32 (2017) 1750139

32 (2017) 1750139

32 (2017) 1750139

32 (2017) 1771001

32 (2017) 1750126

32 (2017) 1750139

32 (2017) 1750139

32 (2017) 1750139

32 (2017) 1750139

32 (2017) 1750139

32 (2017) 1750139

32 (2017) 1750058

32 (2017) 1750139

32 (2017) 1750139

32 (2017) 1750154

32 (2017) 1750217

32 (2017) 1730004

32 (2017) 1750033

32 (2017) 1750123

32 (2017) 1750142

32 (2017) 1750060

32 (2017) 1750139

32 (2017) 1750139

32 (2017) 1750192

32 (2017) 1750139 
Shamir, M. F. \& Ahmad, M., Some exact solutions in $f(\mathcal{G}, T)$ gravity via Noether symmetries

Shankaranarayanan, S., see Bhattacharya

Sharif, M. \& Nawazish, I., Noether symmetries and anisotropic universe models in $f(R, T)$ gravity

Sharif, M. \& Nazir, K., Noncommutative wormhole solutions in $F\left(T, T_{\mathcal{G}}\right)$ gravity

Sharif, M. \& Sadiq, S., Cracking in charged anisotropic cylinder

Sharif, M. \& Siddiqa, A., Interaction of viscous modified Chaplygin gas with $f(R, T)$ gravity

Shears, T., see Aaltonen

Shepard, P. F., see Aaltonen

Shifman, M. \& Vainshtein, A., (In)dependence of $\theta$ in the Higgs regime without axions

Shifman, M. \& Yung, A., FradkinShenker continuity and "insteadof-confinement" phase

Shiltsev, V., Fermilab proton accelerator complex status and improvement plans

Shimo, H., see Cho

Shimojima, M., see Aaltonen

Shimomura, T., see Kobayashi

Shinmura, S., see Ngo

Shiraishi, S., see Aaltonen

Shochet, M., see Aaltonen

Shreyber, I., see Aaltonen

Shu, X.-Y., Huang, Y.-F. \& Zong, H.-S., Gamma-ray bursts generated from phase transition of neutron stars to quark stars

Shu, X.-Y., Huang, Y.-F. \& Zong, H.-S., Conversion of neutron stars to $2+1$ flavor Nambu-JonaLasinio quark stars as a mechanism for gamma-ray bursts

Siddiqa, A., see Sharif

Silva, M., see Alves

Silvestrin, L., see Maggiore

Simonenko, A., see Aaltonen

Sinervo, P., see Aaltonen

Singh, G., see Mali

Singh, Ksh. N., Bhar, P., Rahaman, F., Pant, N. \& Rahaman, M., Conformally non-flat spacetime representing dense compact objects

Singh, P., see Kumar

Singh, S. S., see Jarwal
Sinha, A. K. \& Majumdar, P., Thermal stability of charged rotating quantum black holes

Sinitskaya, A. V., see Korenblit

Sissakian, A., see Aaltonen

Sitarz, M., see Szkliniarz

Skoptsov, M. B., see Kazantsev

32 (2017) 1750136

Sliwa, K., see Aaltonen

Smith, J. R., see Aaltonen

Snider, F. D., see Aaltonen

32 (2017) 1750083

Soha, A., see Aaltonen

Solà, J., Gómez-Valent, A. \& de Cruz

32 (2017) $1750091 \quad$ Pérez, J., Dynamical dark energy: Scalar fields and running vacuum

Somalwar, S., see Aaltonen

32 (2017) 1750151

32 (2017) 1750139

32 (2017) 1750139

Son, H.-J., see Kim

Sondenheimer, R., see Egger

Sorin, V., see Aaltonen

Soylu, A., see Koyuncu

Squillacioti, P., see Aaltonen

32 (2017) 1750084

Srivastava, A. M., see Das

Srivastava, R., see Modak

St. Denis, R., see Aaltonen

32 (2017) 1750159

St. Louis, C., see Matei

Stadnik, Y. V. \& Flambaum, V. V., New generation low-energy probes

32 (2017) 1730012

32 (2017) 1750127

32 (2017) 1750139

32 (2017) 1750142

32 (2017) 1750190

32 (2017) 1750139

32 (2017) 1750139

32 (2017) 1750139 for ultralight axion and scalar dark matter

Staicova, D. \& Stoilov, M., Cosmological aspects of a unified dark energy and dust dark matter model

Stancari, M., see Aaltonen

Stanitzki, M., see Aaltonen

Stecker, F. W., Search for the footprints of new physics with laboratory and cosmic neutrinos

Stelzer, B., see Aaltonen

32 (2017) 1750027

Stelzer-Chilton, O., see Aaltonen

Stentz, D., see Aaltonen

Stepanyantz, K. V., see Kazantsev

Stodolsky, L., Behavior of very high energy hadronic cross-sections

32 (2017) 1750209

32 (2017) 1750151

32 (2017) 1740013

32 (2017) 1740010

32 (2017) 1750139

32 (2017) 1750139

32 (2017) 1750024

32 (2017) 1750093

32 (2017) 1750021

32 (2017) 1750037
Stoilov, M., see Staicova

Stolarz, A., see Szkliniarz

Strologas, J., see Aaltonen

Strycker, G. L., see Aaltonen

Stuchlík, see Toshmatov

Sudo, Y., see Aaltonen

Sukhanov, A., see Aaltonen

Suleymanov, M., see Ali

Sun, W., Physical angular momentum separation for QED

Surette, G., see Matei

Suslov, I., see Aaltonen

Svadkovsky, I., see Belotsky
32 (2017) 1750208

32 (2017) 1750066

32 (2017) 1750139

32 (2017) 1740012

32 (2017) 1750194

32 (2017) 1750139

32 (2017) 1750139

32 (2017) 1750139

32 (2017) 1750139

32 (2017) 1750054

32 (2017) 1750139

32 (2017) 1750203

32 (2017) 1750212

32 (2017) 1750139

32 (2017) 1750050

32 (2017) 1750139

32 (2017) 1750170

32 (2017) 1750004

32 (2017) 1750139

32 (2017) 1740011

32 (2017) 1740004

32 (2017) 1750006

32 (2017) 1750139

32 (2017) 1750139

32 (2017) 1730014

32 (2017) 1750139

32 (2017) 1750139

32 (2017) 1750139

32 (2017) 1750194

32 (2017) 1730028

32 (2017) 1750006

32 (2017) 1740012

32 (2017) 1750139

32 (2017) 1750139

32 (2017) 1775001

32 (2017) 1750139

32 (2017) 1750139

32 (2017) 1750167

32 (2017) 1750063

32 (2017) 1740011

32 (2017) 1750139

32 (2017) 1740008 
Szanyi, I., see Jenkovszky

Szkliniarz, K., Sitarz, M., Jastrzębski, J., Choiński, J., Jakubowski, A., Kapinos, K., Kisieliński, M., Stolarz, A., Trzcińska, A., Wojtkowska, J. \& Zipper, W., Production efficiency and radioisotopic purity of ${ }^{99 \mathrm{~m}} \mathrm{Tc}$ formed using the $(p, 2 n)$ reaction on a highly enriched ${ }^{100}$ Mo target

Tabassam, U., see Ali

Taghavi, R. \& Mirjalili, A., Extracting the QCD $\Lambda_{\overline{\mathrm{MS}}}$ parameter in Drell-Yan process using CollinsSoper-Sterman approach

Tahim, M. O., see Bezerra

Tahim, M. O., see Landim

Takemasa, K., see Aaltonen

Takeuchi, Y., see Aaltonen

Tanaka, S., see Kawamura

Tang, J., see Aaltonen

Tang, Y., Interacting dark matter and dark radiation

Tanuma, R., see Kawamura

Tapia, N., see Das

Tecchio, M., see Aaltonen

Teng, P. K., see Aaltonen

Thom, J., see Aaltonen

Thome, J., see Aaltonen

Thompson, G. A., see Aaltonen

Thomsen, A. E., see Alanne

Thomson, E., see Aaltonen

Tkaczyk, S., see Aaltonen

Tokar, S., see Aaltonen

Tollefson, K., see Aaltonen

Tomura, T., see Aaltonen

Torre, S., see Aaltonen

Torretta, D., see Aaltonen

Toshmatov, B., Stuchlík \& Ahmedov, B., Comments on "Casimir effect in the Kerr spacetime with quintessence"

Totaro, P., see Aaltonen

Totsuka, Y., see Kawamura

Toyoda, T., see Kawamura

Tripathi, B. V., Nandan, H. \& Purohit, K. D., Global monopole in a broken-symmetric theory of gravitation

Troshin, S. M. \& Tyurin, N. E., Analytization of elastic scattering amplitude

Troshin, S. M. \& Tyurin, N. E., Does the diffraction cone shrinkage with energy originate from unitarity?

Trovato, M., see Aaltonen
32 (2017) 1750116



$\mathbf{3 2}(2017) 1740012$
$\mathbf{3 2}(2017) 1750167$

$\mathbf{3 2}(2017) 1750040$
$\mathbf{3 2}(2017) 1750005$
$\mathbf{3 2}(2017) 1750193$
$\mathbf{3 2}(2017) 1750139$
$\mathbf{3 2}(2017) 1750139$
$\mathbf{3 2}(2017) 1750058$
$\mathbf{3 2}(2017) 1750139$
$\mathbf{3 2}(2017) 1740006$
$\mathbf{3 2}(2017) 1750058$
$\mathbf{3 2}(2017) 1750175$
$\mathbf{3 2}(2017) 1750139$
$\mathbf{3 2}(2017) 1750139$
$\mathbf{3 2}(2017) 1750139$
$\mathbf{3 2}(2017) 1750139$
$\mathbf{3 2}(2017) 1750139$
$\mathbf{3 2}(2017) 1750143$
$\mathbf{3 2}(2017) 1750139$
$\mathbf{3 2}(2017) 1750139$
$\mathbf{3 2}(2017) 1750139$

$\mathbf{3 2}(2017) 1750139$

32 (2017) 1775001

32 (2017) 1750139

32 (2017) 1750058

32 (2017) 1750058

32 (2017) 1750061

32 (2017) 1750028

32 (2017) 1750168

32 (2017) 1750139
Trzcińska, A., see Szkliniarz Ttito-Guzmán, P., see Aaltonen Tu, Y., see Aaltonen Tyurin, N. E., see Troshin Tyurin, N. E., see Troshin Ukegawa, F., see Aaltonen Ul Islam, N., see Ganai Ulhoa, S. C., see da Silva Uozumi, S., see Aaltonen Upadhaya, S., see Saha Urakawa, Y., see Kobayashi Vainshtein, A., see Shifman Valuyan, M. A., Radiative correction to the Casimir energy for massive scalar field on a spherical surface van Putten, M. H. P. M., Galaxy rotation curves and the deceleration parameter in weak gravity

Varganov, A., see Aaltonen

Vásquez, Y., see González

Vassilevich, D., see Khodaee Vázquez, F., see Aaltonen

Velev, G., see Aaltonen

Velizhanin, V. N., Generalised double-logarithmic equation in QCD

Vellidis, C., see Aaltonen

Vichi, S., see Cicoria

Vidal, M., see Aaltonen

Vignaroli, N., see Molinaro

Vila, I., see Aaltonen

Vilar, R., see Aaltonen

Visser, M., see Gray

Vizán, J., see Aaltonen

Vogel, M., see Aaltonen

Voloshin, M. B., see Bondar

Volovik, G. E., see Klinkhamer

Volpi, G., see Aaltonen

Vulfs, T. O. \& Guendelman, E. I., Galileon string measure and other modified measure extended objects

Wagner, P., see Aaltonen

Wagner, R. L., see Aaltonen

Wakisaka, T., see Aaltonen

Wallny, R., see Aaltonen

Wang, G.-L., see Li

Wang, Q.-W., see Fan

Wang, S. M., see Aaltonen

Wang, T.-H., see Li

Wang, W.-J., Yan, Z.-H., Guan, R.-H. \& Wei, X.-N., Electric dipole moment induced by CP-violating deformations in the noncommutative Standard Model

Wang, Z.-H., see Li
32 (2017) 1740012

32 (2017) 1750139

32 (2017) 1750139

32 (2017) 1750028

32 (2017) 1750168

32 (2017) 1750139

32 (2017) 1750214

32 (2017) 1750113

32 (2017) 1750139

32 (2017) 1750018

32 (2017) 1750142

32 (2017) 1750084

32 (2017) 1750128

32 (2017) 1730019

32 (2017) 1750139

32 (2017) 1750173

32 (2017) 1750157

32 (2017) 1750139

32 (2017) 1750139

32 (2017) 1750213

32 (2017) 1750139

32 (2017) 1740014

32 (2017) 1750139

32 (2017) 1730024

32 (2017) 1750139

32 (2017) 1750139

32 (2017) 1771001

32 (2017) 1750139

32 (2017) 1750139

32 (2017) 1750025

32 (2017) 1750103

32 (2017) 1750139

32 (2017) 1750211

32 (2017) 1750139

32 (2017) 1750139

32 (2017) 1750139

32 (2017) 1750139

32 (2017) 1750013

32 (2017) 1750107

32 (2017) 1750139

32 (2017) 1750013

32 (2017) 1750044 32 (2017) 1750013 
Warburton, A., see Aaltonen

Watabiki, Y., see J. Ambjørn

Watanabe, Y. X., see Kawamura

Waters, D., see Aaltonen

Wei, X.-N., see Wang

Weinberger, M., see Aaltonen

Weiner, M., see Matolcsi

Wen, W.-Y., see Hsu

West, P., On the different formulations of the $E_{11}$ equations of motion

Whitehouse, B., see Aaltonen

Whiteson, D., see Aaltonen

Wick, F., see Aaltonen

Wicklund, E., see Aaltonen

Wilbur, S., see Aaltonen

Williams, H. H., see Aaltonen

Wilson, J. S., see Aaltonen

Wilson, P., see Aaltonen

Winer, B. L., see Aaltonen

Wittich, P., see Aaltonen

Wojtkowska, J., see Szkliniarz

Wolfe, H., see Aaltonen

Wright, T., see Aaltonen

Wu, Q., see He

Wu, X., see Aaltonen

Wu, Y., see Lin

Wu, Z., see Aaltonen

Wu, Z. Q., Ping, J. L. \& Zong, H. S., Effects of Fierz transformation on gap equation and CEP at finite chemical potential and finite temperature in Hartree-Fock approximation

Xi, P., Jing, Y.-L., Lü, W.-D. \& Zhai, X.-H., Statefinder diagnostic for a generalized Proca model

Xie, J. \& Peng, H., Predictions of $\rho$ meson cross-sections between AdS/QCD and boosted Gaussian wave functions in the color glass condensate model

Xing, Z. Z. \& Zhao, Z. H., Neutrinoless double-beta decays: New insights

Xiu, W.-T., see Gao

Yagi, J., Branes and integrable lattice models

Yamamoto, K., see Aaltonen

Yamaoka, J., see Aaltonen

Yan, Y., see Zhao

Yan, Z.-H., see Wang

Yanar, H., see Salti

Yang, C. N., Jeremy Bernstein's monologue
32 (2017) 1750139

32 (2017) 1750224

32 (2017) 1750058

32 (2017) 1750139

32 (2017) 1750044

32 (2017) 1750139

32 (2017) 1750147

32 (2017) 1750145

32 (2017) 1750096

32 (2017) 1750139

32 (2017) 1750139

32 (2017) 1750139

32 (2017) 1750139

32 (2017) 1750139

32 (2017) 1750139

32 (2017) 1750139

32 (2017) 1750139

32 (2017) 1750139

32 (2017) 1750139

32 (2017) 1740012

32 (2017) 1750139

32 (2017) 1750139

32 (2017) 1750052

32 (2017) 1750139

32 (2017) 1750188

32 (2017) 1750139

32 (2017) 1750222

32 (2017) 1750160

32 (2017) 1750081

32 (2017) 1730011 32 (2017) 1750072

32 (2017) 1730003

32 (2017) 1750139

32 (2017) 1750139

32 (2017) 1750051

32 (2017) 1750044

32 (2017) 1750183

32 (2017) 1730017
Yang, Q., Axions and dark matter

Yang, T., see Aaltonen

Yang, U. K., see Aaltonen

Yang, X.-Y., see Gao

Yang, Y. C., see Aaltonen

Yao, W.-M., see Aaltonen

Yeh, G. P., see Aaltonen

Yi, K., see Aaltonen

Yi, S.-X., On gravitational waveCherenkov radiation from photons when passing through diffused dark matters

Yoh, J., see Aaltonen

Yoon, Y., Maxwell-Boltzmann type Hawking radiation

Yorita, K., see Aaltonen

Yoshida, T., see Aaltonen

Yousaf, Z., Ilyas, M. \& Bhatti, M. Z., Influence of modification of gravity on spherical wormhole models

Yousaf, Z., see Bhatti

Yu, G. B., see Aaltonen

Yu, H. \& Ma, B.-Q., Unification of gravity and quantum field theory from extended noncommutative geometry

Yu, I., see Aaltonen

Yu, S. S., see Aaltonen

Yuan, L., see Zhang

Yue, C.-X., see Lai

Yun, J. C., see Aaltonen

Yung, A., see Shifman

Zaeem-ul-Haq Bhatti, M., Anwar, A. \& Ashraf, S., Construction of thin shell wormholes from metric $f(R)$ gravity

Zagni, F., see Cicoria

Zanetti, A., see Aaltonen

Zare, S., see Hassanabadi

Zeng, Y., see Aaltonen

Zhai, X.-H., see Xi

Zhang, J. \& Yuan, L., Resonant parameters of $Y(4390)$

Zhang, L., Gao, Y., Zheng, H., Huang, M. R. \& Liu, X., Moments of the three-parameter Fermi distribution

Zhang, Y., Chan, A. H. \& Oh, C. H., Stochastic model for supersymmetric particle branching process Zhang, Y., see Dev

Zhao, T., Li, C.-M., Zhao, Y.-P., Yan, Y., Luo, X.-L. \& Zong, H.-S., A phenomenological study of hybrid stars in which the cross-over
32 (2017) 1740003

32 (2017) 1750139

32 (2017) 1750139

32 (2017) 1750072

32 (2017) 1750139

32 (2017) 1750139

32 (2017) 1750139

32 (2017) 1750139

32 (2017) 1750059

32 (2017) 1750139

32 (2017) 1750071

32 (2017) 1750139

32 (2017) 1750139

32 (2017) 1750163

32 (2017) 1750042

32 (2017) 1750139

32 (2017) 1750030

32 (2017) 1750139

32 (2017) 1750139

32 (2017) 1750184

32 (2017) 1750039

32 (2017) 1750139

32 (2017) 1750159

32 (2017) 1750111

32 (2017) 1740014

32 (2017) 1750139

32 (2017) 1750085

32 (2017) 1750139

32 (2017) 1750160

32 (2017) 1750184

32 (2017) 1750195

32 (2017) 1750020

32 (2017) 1740007 
transition from quark to hadron makes the EOS stiffer in contrast to the hybrid EOS based on Maxwell condition

Zhao, Y., Obtaining nonvanishing $\theta_{13}$ with constrained neutrino Yukawa matrix and implications for flavor model buildings

Zhao, Y.-J., Hawking radiation from the holographic screen

Zhao, Y.-P., see Zhao

Zhao, Z. H., see Xing

Zheltukhin, A. A., Inflation versus collapse in brane matter

\begin{tabular}{|c|c|}
\hline \multirow{6}{*}{32 (2017) 1750051} & Zheng, H., see Zhang \\
\hline & Zipper, W., see Szkliniarz \\
\hline & Zong, H.-S., see Shu \\
\hline & Zong, H.-S., see Zhao \\
\hline & Zong, H.-S., see Fan \\
\hline & Zong, H.-S., see Shu \\
\hline & Zong, H. S., see Wu \\
\hline 32 (2017) 1750080 & $\begin{array}{l}\text { Zotov, A., Relativistic elliptic matrix } \\
\text { tops and finite Fourier transforma- }\end{array}$ \\
\hline 32 (2017) 1750162 & tions \\
\hline 32 (2017) 1750051 & Zucchelli, S., see Aaltonen \\
\hline 32 (2017) 1730011 & $\begin{array}{l}\text { Zyryanova, O. V., Composite opera- } \\
\text { tors in functional renormalization }\end{array}$ \\
\hline 32 (2017) 1750199 & group approach \\
\hline
\end{tabular}

32 (2017) 1750195

32 (2017) 1740012

32 (2017) 1750027

32 (2017) 1750051

32 (2017) 1750107

32 (2017) 1750209

32 (2017) 1750222

32 (2017) 1750169

32 (2017) 1750139

32 (2017) 1750153 